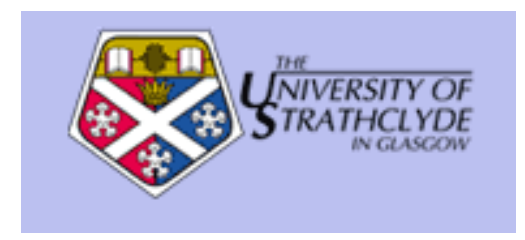

Manaoch, E. and Trendafilova, I. (2010) Damage detections in nonlinear vibrating thermally loaded plates. In: Materials With Complex Behaviour. Advanced Structural Materials . Springer Verlag, Berlin Heidelberg, Ch 14. ISBN 978-3-642-12667-3

http://strathprints.strath.ac.uk/25575/

Strathprints is designed to allow users to access the research output of the University of Strathclyde. Copyright (C) and Moral Rights for the papers on this site are retained by the individual authors and/or other copyright owners. You may not engage in further distribution of the material for any profitmaking activities or any commercial gain. You may freely distribute both the url (http://strathprints.strath.ac.uk) and the content of this paper for research or study, educational, or not-for-profit purposes without prior permission or charge. You may freely distribute the url (http://strathprints.strath.ac.uk) of the Strathprints website.

Any correspondence concerning this service should be sent to The Strathprints Administrator: eprints@cis.strath.ac.uk 


\title{
Damage Detections in Nonlinear Vibrating Thermally Loaded Plates
}

\author{
E. Manoach and I. Trendafilova
}

\section{Introduction}

The main objective of structural health monitoring (SHM) is to ascertain whether damage is present or not in a structure. Most vibration-based structural health monitoring methods (VSHM) are based on the fact that damage will alter the stiffness, mass or energy dissipation properties of a structure which in turn will alter its measured vibration response.

These methods are widely used for structural health monitoring and damage assessment purposes. Their application is somewhat limited by the need of a precise enough model of the structural vibration response. If some nonlinearities or environmental conditions (like the elevated temperature, for example) are not taken into account in the model, then a model-based VSHM method could give a false alarm due to a discrepancy between the measured and the modelled response. Temperature changes can and do affect substantially the vibration response of a structure. Thermal loads introduce stresses due to thermal expansion, which lead to changes in the modal properties. Thermal loads can also cause buckling and in some cases even lead to chaotic behaviour [1-5].

Thus, on a lot of occasions the presence of a temperature field can either mask the effect of damage or increase it, which will render a VSHM method ineffective it might give no alarm when a fault is present or give a false alarm. This is why it is vital to be able to take into account the temperature changes when developing VSHM procedures.

Most of the previous efforts of researchers in the area of VSHM were directed towards methods based on linear modal analysis [6-10]. One of the main problems with these methods comes from the fact that in general damage starts as a local phenomenon and does not necessarily affect significantly the modal characteristics of the structure. In many cases the lower order resonance frequencies and mode shapes are not very sensitive to damage, except in cases of very large damage

E. Manoach $(\bowtie)$

Institute of Mechanics, Bulgarian Academy of Sciences, 1113 Sofia, Bulgaria 
$[6,11]$. Thus in reality it may be difficult to distinguish if damage is indeed the reason behind, e.g., a decrease in frequency or it is caused by environmental or operational conditions changes.

Many VSHM methods are inherently limited to linear systems - they use, for example, the superposition principle in the analysis - and cannot account for the effects of non-linearities. Another problem with a number of VSHM methods is that they rely on a linear model of the structure. As the theoretical model itself can only approximate the actual behaviour of the vibrating structure, it will introduce computational errors [6]. These errors will be greater if the non-linearities of the system are substantial. Since they are not taken into account in the model such methods might give false alarms due to a discrepancy between the measured and the modelled/expected response.

To address some of the above mentioned problems, new concepts in vibrationbased monitoring have been emerging recently. These employ measured time series of the structural vibration response, or, often concomitantly, non-linear systems theory. Most of the studies in this field are devoted to the extraction of features from the structural vibration response, which can indicate the presence of damage and its location. In [12] the authors use the beating phenomenon for damage detection purposes. In [13] and [14] new attractor-based metrics are introduced as damage sensitive features. The results are promising. In [15] a panel forced by aerodynamic loads and undergoing limit-cycle oscillations and chaos is investigated. The von Kármán strain displacement relation is employed and a model of the system constituted by ordinary differential equations of motion is achieved by employing finite differences. The upstream endpoint of the panel has been considered supported by a spring of variable stiffness. Changes in the stiffness of a spring have been detected by exploring the chaotic dynamics of the panel.

In [16] a possibility for representing, interpreting and visualising the vibration response of vibrating panels using time domain measurements is investigated. The panels are thin orthotropic plates and are modelled by finite elements. It was found that the first ten resonant frequencies show low sensitivity to damage. Then the simulated vibration response of the panel is transformed and expanded in a new phase space. Preliminary results suggest that it should be possible to use the distribution of points on the attractor to extract damage sensitive features.

In our previous works [11] and [17] a numerical approach to study the geometrically non-linear vibrations of rectangular plates with and without damage is developed. A damage index and a method for damage detection and location, based on the Poincaré map of the response, have been proposed. The suggested damage assessment method shows good capability to detect and localize damage in plates.

Although the approach seems to hold a lot of potential, there is limited research addressing VSHM methods based on time series analysis and non-linear dynamics.

The main objectives of this study are twofold: (i) to study the influence of defects, elevated temperatures and their combination on the dynamic characteristics of the plate and on its geometrically nonlinear dynamic response; (ii) to test the criteria for identification of irregularities (defects) in structures proposed in $[11,17]$ taking into 
account the elevated temperature by analyzing the Poincaré map of the structural vibration response.

The application of the proposed approach is demonstrated on rectangular plates with defects at elevated temperatures. The temperature is assumed uniformly distributed over the plate surface and thickness. The plates are subjected to a harmonic loading which leads to large amplitude vibrations. The influence of damage on the time-history diagrams of the plate, as well as on the geometry of its phase-space is studied. A VSHM method is developed which applies a criterion based on features sensitive to temperature changes and damage in the same time. These features use the Poincaré maps of the structural vibration response. Taking into account the temperature influence on the extracted features allows the detection of damage and shows its location for structures subjected to temperature changes. The proposed study demonstrates the importance of taking into account the correct/exploitation temperature in a damage detection process. It is shown that in some cases of elevated temperature the Poincaré maps based criterion may be unsuitable.

\section{Theoretical Model}

The object of the investigation is a rectangular plate with sides $a$ and $b$ and thickness $h$, subjected to temperature changes and a dynamic loading $p(x, y, t)$ perpendicular to the plate (Fig. 1a). The geometrically nonlinear version of the Mindlin plate theory is used to model the plate behaviour, so that the shear deformation and rotatory inertia are taken into account. At each point of the middle surface of the plate, the displacements in the $x, y, z$ directions are denoted by $u, v, w$, respectively, $\psi_{x}(x, y, t)$ and $\psi_{y}(x, y, t)$ are the angles of the rotation of the normal of the cross section to the plate mid-plane (see Fig. 1b).
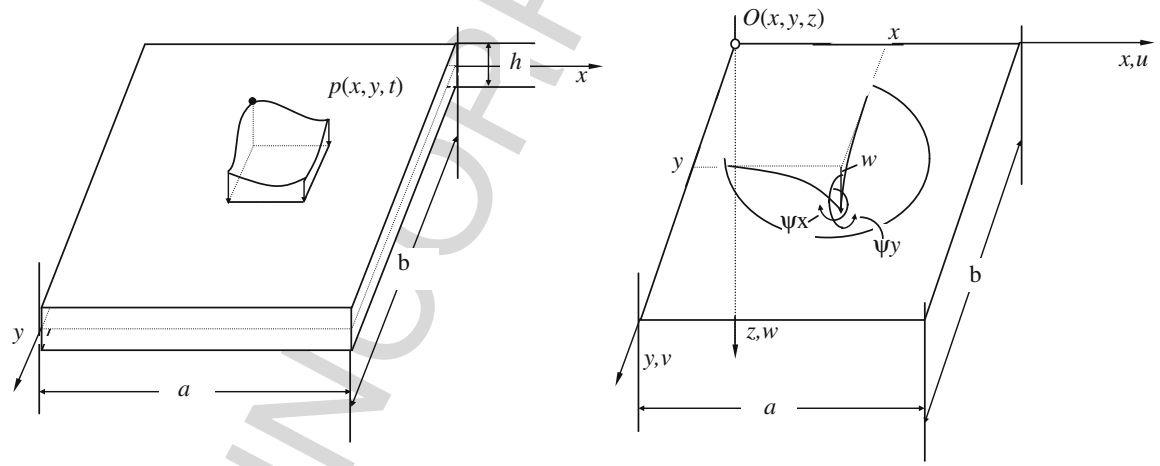

Fig. 1 Plate geometry and coordinate system. (a) Plate dimensions and loading. (b) Mid-plane of the plate and the components of the generalized displacement vector 
The presence of a defect can be modelled as a reduction of the plate thickness or a stiffness reduction and therefore a variation of the flexural rigidity in the governing equations is used. The basic equations of the plate motion are described below.

\subsection{Geometrical Relationships}

The strain and curvature-displacements relationships associated with the mid-plane of the plate for large displacements and shear can be expressed as:

$$
\begin{gathered}
\varepsilon_{x}^{0}=\frac{\partial u}{\partial x}+\frac{1}{2}\left(\frac{\partial w}{\partial x}\right)^{2}, \varepsilon_{y}^{0}=\frac{\partial v}{\partial y}+\frac{1}{2}\left(\frac{\partial w}{\partial y}\right)^{2}, \varepsilon_{x y}^{0}=\frac{\partial u}{\partial y}+\frac{\partial v}{\partial x}+\frac{\partial w}{\partial x} \frac{\partial w}{\partial y}, \\
\varepsilon_{x z}^{0}=\psi_{x}+\frac{\partial w}{\partial x}, \varepsilon_{y z}^{0}=\psi_{y}+\frac{\partial w}{\partial y} \\
k_{x}^{0}=\frac{\partial \psi_{x}}{\partial x}, k_{y}^{0}=\frac{\partial \psi_{y}}{\partial y}, k_{x y}^{0}=\frac{\partial \psi_{x}}{\partial y}+\frac{\partial \psi_{y}}{\partial x}
\end{gathered}
$$

and the strain vector is given by:

$$
\varepsilon=\left\{\varepsilon_{x}^{0}+z k_{x}^{0}, \varepsilon_{y}^{0}+z k_{y}^{0}, \varepsilon_{x y}^{0}+z k_{x y}^{0}, f(z) \varepsilon_{x z}^{0}, f(z) \varepsilon_{y z}^{0}\right\}^{T}
$$

where $f(z)$ is a function describing the distribution of the shear strain along the plate thickness.

\subsection{Constitutive Equations}

Assuming that the material of the plate is linear elastic and isotropic the relations for the stress and strain components are given by:

$$
\begin{gathered}
\sigma_{x}=\frac{\boldsymbol{E}(\boldsymbol{x}, \boldsymbol{y})}{1-v^{2}}\left[\varepsilon_{x}+v \varepsilon_{y}\right]-\frac{\boldsymbol{E}(\boldsymbol{x}, \boldsymbol{y})}{1-v} \alpha_{T} \Delta \boldsymbol{T}, \\
\sigma_{y}=\frac{\boldsymbol{E}(\boldsymbol{x}, \boldsymbol{y})}{1-v^{2}}\left[\varepsilon_{y}+v \varepsilon_{x}\right]-\frac{\boldsymbol{E}(\boldsymbol{x}, \boldsymbol{y})}{1-v} \alpha_{T} \Delta \boldsymbol{T}, \\
\sigma_{x z}=\boldsymbol{n}^{2} \boldsymbol{G} \varepsilon_{x z}, \quad \sigma_{y z}=\boldsymbol{n}^{2} \boldsymbol{G} \varepsilon_{y z}
\end{gathered}
$$

In terms of generalized stresses the above equations take the form:

$$
N_{x}=A\left(\varepsilon_{x}^{0}+\nu \varepsilon_{y}^{0}\right)-A \alpha_{T} \gamma^{T}, N_{y}=A\left(\varepsilon_{y}^{0}+\nu \varepsilon_{x}^{0}\right)-A \alpha_{T} \gamma^{T}, N_{x y}=\frac{1-v}{2} A \varepsilon_{x y}^{0}
$$


Damage Detections in Nonlinear Vibrating Thermally Loaded Plates

$$
\begin{aligned}
& M_{x}=D\left(\kappa_{x}^{o}+v \kappa_{y}^{o}\right)-A \alpha_{T} \boldsymbol{\kappa}^{T}, M_{y}=D\left(\boldsymbol{\kappa}_{y}^{0}+v \boldsymbol{\kappa}_{x}^{0}\right)-A \alpha_{T} \boldsymbol{\kappa}^{T}, M_{x y}=\frac{1}{2}(1-v) D \boldsymbol{\kappa}_{x y}^{0}, \\
& Q_{x}=\frac{1}{2}(1-v) n^{2} A \varepsilon_{x z}^{0}, \quad Q_{y}=\frac{1}{2}(1-v) n^{2} A \varepsilon_{y z}^{0} .
\end{aligned}
$$

where

$$
\begin{aligned}
\gamma^{T}(x, y) & =\int_{-h / 2}^{h / 2} \Delta T(x, y, z) d z, \quad \kappa^{T}(x, y)=\int_{-h / 2}^{h / 2} \Delta T(x, y, z) z d z, \\
A(x, y) & =\frac{E(x, y) h(x, y)}{1-v^{2}}, \quad D(x, y)=\frac{A(x, y) h(x, y)^{2}}{12}
\end{aligned}
$$

In Eqs. (3), (4) and (5) $E$ is the Young modulus, $v$ is the Poison ratio, $N_{x}, N_{y}$ and $N_{x y}$ are the stress resultants in the mid-plane of the plate, $M_{x}, M_{y}$ and $M_{x y}$ are the stress couples and $Q_{x}$ and $Q_{y}$ are the transverse shear stress resultants, $\alpha_{T}$ is the coefficient of thermal expansion and $\Delta T$ (Kelvin) is the temperature variation (in general it can be assumed non-uniform along the plate length and thickness) with respect to a reference temperature. $n^{2}$ is a shear correction factor which is assumed equal to $5 / 6$ throughout the paper.

\subsection{Equations of Motion}

The equilibrium equations may be deducted by considering the conditions for translational equilibrium in the $x, y$ and $z$ directions and for rotational equilibrium about $x$ and $y$. They are as follows:

$$
\begin{gathered}
\frac{\partial N_{x}}{\partial x}+\frac{\partial N_{x y}}{\partial y}+\rho h \ddot{u}_{x}=0 \\
\frac{\partial N_{y}}{\partial y}+\frac{\partial N_{x y}}{\partial x}+\rho h \ddot{u}_{y}=0 \\
\frac{\partial M_{x}}{\partial x}+\frac{\partial M_{x y}}{\partial y}-Q_{x}+c_{2} \frac{\partial \psi_{x}}{\partial t}+\frac{\rho h^{3}}{12} \ddot{\psi}_{x}=0 \\
\frac{\partial M_{y}}{\partial y}+\frac{\partial M_{x y}}{\partial x}-Q_{y}+c_{2} \frac{\partial \psi_{y}}{\partial t}+\frac{\rho h^{3}}{12} \ddot{\psi}_{y}=0 \\
\frac{\partial Q_{x}}{\partial x}+\frac{\partial Q_{y}}{\partial y}+N_{x} \frac{\partial^{2} w}{\partial x^{2}}+N_{y} \frac{\partial^{2} w}{\partial y^{2}}+2 N_{x y} \frac{\partial^{2} w}{\partial x \partial y}+c_{1} \frac{\partial w}{\partial t}+\rho h \ddot{w}=-p
\end{gathered}
$$

Here and throughout in the paper dots over variables represents derivation with respect to time, $c_{1}$ and $c_{2}$ denote the damping coefficients, and $\rho$ is the density of the plate material. 


\subsection{Boundary and Initial Conditions}

In the present work fully clamped plates, i.e. plates for which all their four edges are clamped and in-plane fixed, are considered. This means that all displacements $u, v$ and $w$ and angular rotations $\psi_{x}$ and $\psi_{y}$ are zero along the boundaries. The influence of the temperature variation is more essential for such plates due to the thermal expansion.

The initial conditions are accepted in the following general form:

$$
\begin{aligned}
w(x, y, 0) & =w^{0}(x, y), \quad \dot{w}(x, y, 0)=\dot{w}^{0}(x, y), \\
\psi_{x}(x, y, 0) & =\psi_{x}^{0}(x, y), \dot{\psi}_{y}(x, y, 0)=\dot{\psi}_{y}^{0}(x, y,), x \in[0, a], y \in[0, b]
\end{aligned}
$$

\section{Solution of the Problem}

\subsection{Reorganizing the Equations of the Plate Motion}

The equation of motions (6) can be rewritten in the following form:

$$
\begin{gathered}
\frac{\partial}{\partial x}\left[A\left(\frac{\partial u}{\partial x}+v \frac{\partial v}{\partial y}\right)\right]+\frac{\partial}{\partial y}\left[\frac{(1-v) A}{2}\left(\frac{\partial u}{\partial y}+\frac{\partial v}{\partial x}\right)\right]+\rho h \ddot{u}=G_{u}+G_{u}^{T} \\
\frac{\partial}{\partial y}\left[A\left(\frac{\partial v}{\partial y}+v \frac{\partial u}{\partial x}\right)\right]+\frac{\partial}{\partial x}\left[\frac{A(1-v)}{2}\left(\frac{\partial v}{\partial x}+\frac{\partial u}{\partial y}\right)\right]+\rho h \ddot{v}=G_{v}+G_{v}^{T} \quad(8 \mathrm{a}-\mathrm{e}) \\
\frac{\partial}{\partial x}\left(D\left[\frac{\partial \psi_{x}}{\partial x}+v \frac{\partial \psi_{y}}{\partial y}\right]\right)+\frac{(1-v)}{2} \frac{\partial}{\partial y}\left(D\left[\frac{\partial \psi_{x}}{\partial y}+v \frac{\partial \psi_{y}}{\partial x}\right]\right) \\
-\frac{\left(1-v^{2}\right) n^{2} A}{2}\left(\psi_{x}+\frac{\partial w}{\partial x}\right)+c_{2} \dot{\psi}_{x}+\frac{\rho h^{3}}{12} \ddot{\psi}_{x}=G_{1}^{T} \\
\frac{\partial}{\partial y}\left(D\left[\frac{\partial \psi_{y}}{\partial y}+v \frac{\partial \psi_{x}}{\partial x}\right]\right)+\frac{(1-v)}{2} \frac{\partial}{\partial x}\left(D\left[\frac{\partial \psi_{y}}{\partial x}+v \frac{\partial \psi_{x}}{\partial y}\right]\right) \\
-\frac{\left(1-v^{2}\right) n^{2} A}{2}\left(\psi_{y}+\frac{\partial w}{\partial y}\right)+c_{2} \dot{\psi}_{y}+\frac{\rho h^{3}}{12} \ddot{\psi}_{y}=G_{2}^{T} \\
\frac{(1-v) n^{2}}{2}\left\{\frac{\partial}{\partial x}\left(A\left[\psi_{x}+\frac{\partial w}{\partial x}\right]\right)+\frac{\partial}{\partial y}\left(A\left[\psi_{y}+\frac{\partial w}{\partial y}\right]\right)\right\}+c_{1} \frac{\partial w}{\partial t}+\rho h \ddot{w} \\
=-p+G^{L}+G_{3}^{T}
\end{gathered}
$$

where

$$
G_{u}=-0.5 \frac{\partial}{\partial x}\left\{A\left[\left(\frac{\partial w}{\partial x}\right)^{2}+\left(\frac{\partial w}{\partial y}\right)^{2}\right]\right\}-0.5 \frac{\partial}{\partial y}\left\{A(1-v)\left(\frac{\partial w}{\partial x} \frac{\partial w}{\partial y}\right)\right\}
$$




$$
\begin{gathered}
G_{v}=-0.5 \frac{\partial}{\partial y}\left\{A\left[\left(\frac{\partial w}{\partial x}\right)^{2}+\left(\frac{\partial w}{\partial y}\right)^{2}\right]\right\}-0.5 \frac{\partial}{\partial x}\left\{A(1-v)\left(\frac{\partial w}{\partial x} \frac{\partial w}{\partial y}\right)\right\} \\
G_{1}^{T}=A(1+v) \alpha_{T} \frac{\partial \kappa_{T}}{\partial x}, \quad G_{2}^{T}=A(1+v) \alpha_{T} \frac{\partial \kappa_{T}}{\partial y}, G_{3}^{T}=A \alpha_{T} \gamma_{T}\left(\frac{\partial^{2} w}{\partial x^{2}}+\frac{\partial^{2} w}{\partial y^{2}}\right) \\
G^{L}(x, y, t)=-\left(N_{x} \frac{\partial^{2} w}{\partial x^{2}}+N_{y} \frac{\partial^{2} w}{\partial x^{2}}+2 N_{x y} \frac{\partial^{2} w}{\partial x \partial y}\right)
\end{gathered}
$$

In this work, only a uniformly distributed temperature field along the plate length and thickness will be considered. Also, it is assumed that the plate gets the elevated temperature instantly. This assumptions leads to settings $G_{1}^{T}=0, G_{2}^{T}=0$.

\subsection{Numerical Approach}

The pseudo-load mode superposition method (PLMS) [2,11, 18-21] is applied to solve the problem for nonlinear vibration of plates. It will be only briefly presented here.

The widely accepted assumption for transversally loaded clamped plates that mid-plane inertia effects are negligible is assumed, i.e. $\rho h \ddot{u}_{x}=\rho h \ddot{u}_{y}=0$. The finite element method is used to discretize the plate equations with respect to the space variables and by using the PLMS they are transformed in the frequency domain. Then an iterative procedure with respect to time is applied for the solution of the obtained system of ordinary differential equations. It is out of the scope of this paper to concentrate on the details of the solution method and the reader is referred to the above mentioned papers [2,18-21] where the method is applied for undamaged plates and in [11] - for damaged ones. Thus the solution procedure will be presented only in brief:

Assuming $G^{u}$ and $G^{v}$ are known functions, Eq. (8a-b) form a linear system of PDEs which can be solved numerically. The left hand sides of Eq. (8c-e) contain only linear terms and therefore the mode superposition method can be used for their solution. Thus, the generalized displacements vector $\mathbf{U}=\left\{\beta \psi_{x}, \beta \psi_{y}, w\right\}^{T}(\beta=$ $\left.h^{2} / 12\right)$ is expanded as a sum of the product of the vectors of the pseudo-normal modes $\mathbf{U}_{n}$ and the time dependent functions $q_{n}(t)$ as follows:

$$
\mathbf{U}=\sum_{n=1}^{N_{f}} \mathbf{U}_{n}(x, y) q_{n}(t) .
$$

Substituting Eq. (10) into Eq. (8c-e), multiplying by $\mathbf{U}_{m}(\mathrm{x}, \mathrm{y})$, integrating the product over the plate surface, invoking the orthogonallity condition, and assuming 
"proportional damping" in the sense $\iint\left(c_{2}\left(\psi_{x n}^{2}+\psi_{y n}^{2}\right)+c_{1} w_{n}^{2}\right) d x d y=2 \xi_{n} \omega_{n}$, the equations for $q n(t)$ will be "uncoupled" in the form:

$$
\ddot{q}_{n}(t)+2 \xi_{n} \omega_{n} \dot{q}_{n}+\omega_{n}^{2} q_{n}(t)=F_{n}(t),
$$

where $\omega_{n}$ are the natural frequencies of the linear elastic (undamped) Mindlin plate, $\xi_{n}$ are the modal damping parameters and

$$
\begin{gathered}
F_{n}(t)=\iint \mathbf{U}_{n}^{T}\left[\mathbf{P}(x, y, t)+\mathbf{G}_{L}(x, y, t)+\mathbf{G}_{T}(x, y, t)\right] d x d y, \\
\mathbf{P}(x, y, t)=(0,0,-p)^{T}, \quad \mathbf{G}_{L}(x, y, t)=\left(0,0, G_{3}^{L}\right)^{T}, \quad \mathbf{G}_{T}(x, y, t)=\left(0,0, G_{3}^{T}\right)^{T} .
\end{gathered}
$$

The initial conditions defined by Eq. (7) are transformed also in terms of $q_{n}(0)$ and $\dot{q}_{n}(0)$ :

$$
\begin{gathered}
q_{n}(0)=q_{n}^{0}, \quad \dot{q}_{n}(0)=\dot{q}_{n}^{0}, \\
q_{n}^{0}=\iint\left(w^{0} w_{n}+\beta \psi_{x}^{0} \psi_{x n}+\beta \psi_{y}^{0} \psi_{y n}\right) d x d y, \\
\dot{q}_{n}^{0}=\iint\left(\dot{w}^{0} \dot{w}_{n}+\beta \dot{\psi}_{x}^{0} \dot{\psi}_{x n}+\beta \dot{\psi}_{y}^{0} \dot{\psi}_{y n}\right) d x d y
\end{gathered}
$$

Using the methodology developed by Kukreti and Issa [18] the pseudo-load vector $\{\mathbf{P}+\mathbf{G}\}$ is interpolated by a quadratic time dependent polynomial, i.e.

$$
\mathbf{P}(x, y, \tau)+\mathbf{G}(x, y, \tau)=\mathbf{A}(x, y)+\mathbf{B}(x, y) \tau+\mathbf{C}(x, y) \tau^{2}, 0 \leq \tau \leq L_{t}
$$

Where $L_{t}=t_{i+1}-t_{i}$ represents the time increment, and $\tau$ which is defined as $\tau=t-t_{i}$, identifies a new time origin for each time increment.

Denoting

$$
\begin{aligned}
& \mathbf{P}_{0}(x, y)=\mathbf{P}(x, y, 0), \mathbf{P}_{1}(x, y)=\mathbf{P}\left(x, y, m L_{t}\right), \mathbf{P}_{2}(x, y)=\mathbf{P}\left(x, y, L_{t}\right), \\
& \mathbf{G}_{0}(x, y)=\mathbf{G}(x, y, 0), \mathbf{G}_{1}(x, y)=\mathbf{G}\left(x, y, m L_{t}\right), \mathbf{G}_{2}(x, y)=\mathbf{G}\left(x, y, L_{t}\right), \\
& 0<m<1,0<x<a, 0<y<b
\end{aligned}
$$

the expressions for the constant yectors $\mathbf{A}, \mathbf{B}$ and $\mathbf{C}$ are derived in terms of $\mathbf{P}_{\mathrm{i}}$ and $\mathbf{G}_{\mathrm{i}}(\mathrm{I}=1$ to 3$)$. The general solution of Eq. (11) is given by:

$$
q_{n}(\tau)=E_{1 n} q_{n}^{0}+E_{2 n} \dot{q}_{n}^{0}+F_{1 n} a_{n}+F_{2 n} b_{n}+F_{3 n} c_{n}
$$

where $E_{1 n}, E_{2 n}, F_{1 n}, F_{2 n}, F_{3 n}$ denote complicated mathematical expressions containing $\omega_{n}, \xi_{n}$ and $\tau$ (see [19]) and

$$
a_{n}=\iint \mathbf{U}_{n}^{T} \mathbf{A}_{n} d x d y, b_{n}=\iint \mathbf{U}_{n}^{T} \mathbf{B}_{n} d x d y, c_{n}=\iint \mathbf{U}_{n}^{T} \mathbf{C}_{n} d x d y
$$


The iteration procedure applied to solve the above Eq. (11) is identical to the ones for circular plates and beams given in [21].

\section{Damage Identification Technique}

There are a lot of techniques to treat the nonlinear structural vibration response in the time domain. The state (phase)-space representation of the structural vibration response is a suitable and powerful tool for studying the dynamic behaviour of a structure. A standard technique for dealing with phase space $(w, \dot{w}, t)$ of periodically driven oscillators is to study the projection of $(w, \dot{w})$ at moments in time $t$, where $t$ is a multiple of the period $T=2 \pi / \omega$. Here $\omega$ can be the frequency of the excitation of the mechanical system, an eigen frequency of the structure, or its multiple, and $T$ is a period of the forcing function, an eigen period of the system, or its multiple. The result of inspecting the phase projection $(w, \dot{w})$ only at specific times $t=k T$ is a sequence of dots, representing the so-called Poincaré map. The steadystate converging trajectories, which represent the attractor, are usually formed in the phase space and in many cases of nonlinear systems they are very sensitive to any changes in the system.

In papers $[11,17]$ the following damage index based on the analysis of the Poincaré map was introduced:

$$
I_{i}^{d}=\frac{S_{i}^{u}-S_{i}^{d}}{S_{i}^{u}},
$$

where

$$
\begin{aligned}
& S_{i}^{u}=\sum_{j=1}^{N_{p}-1} \sqrt{\left(w_{i, j+1}^{u}-w_{i, j}^{u}\right)^{2}+\left(\dot{w}_{i, j+1}^{u}-\dot{w}_{i, j}^{u}\right)^{2}} \\
& S_{i}^{d}=\sum_{j=1}^{N_{p}-1} \sqrt{\left(w_{i, j+1}^{d}-w_{i, j}^{d}\right)^{2}+\left(\dot{w}_{i, j+1}^{d}-\dot{w}_{i, j}^{d}\right)^{2}}
\end{aligned}
$$

In these equations $I=1,2 \ldots N_{\text {nodes }}, N_{\text {node }}$ is the number of nodes, $N_{p}$ is the number of points in the Poincaré map and $\left(w_{i j}^{u}, \dot{w}_{i j}^{u}\right)$ and $\left(w_{i j}^{d}, \dot{w}_{i j}^{d}\right)$ denote the $j$ th point on the Poincaré maps of the undamaged and the damaged states, respectively.

A small (close to 0 ) damage index will indicate no damage, while a big damage index will indicate the presence of a fault at the corresponding location. The above damage index depends on the location of the point on the plate, and consequently it is a function of the plate coordinates $x$ and $y$. One can expect that the maxima of the surface $I^{d}\left(x_{d}, y_{d}\right)(18 \mathrm{a})$ will represent the locations of the damage, i.e. $I_{\max }^{d}\left(x_{d}, y_{d}\right)=$ $\max _{i}\left\{I_{i}^{d}\right\}$. 
The damage criterion based on this index presumes setting a threshold value $T^{d}$ for the damage index and if

$$
I^{d}(x, y)>T^{d}
$$

then one can conclude that the plate is damaged and the areas of points $(\mathrm{x}, \mathrm{y})$ for which Eq. (20) is fulfilled, form the damaged area (areas).

In the present work we shall use the same damage index and damage criterion but taking into account the temperature changes as well, $I^{d}=I^{d}(x, y, \Delta T)$. This suggestion presumes that the damage index defined by Eqs. (18) and (19) is calculated for equal values of $\Delta T$ for the healthy and damaged plate.

\section{Results and Discussions}

Numerical calculation of the vibrational displacements of the healthy and the damaged rectangular plates subjected to mechanical and thermal loading were performed.

The damage was modelled as a reduction (up to 50\%) of the plate thickness in small parts of the plate.

The first example concerns the same plate as the one considered in [1]. The plate has the following dimensions and material properties: $a=0.25 \mathrm{~m}, b=0.24 \mathrm{~m}$, $h=0.00027 \mathrm{~m}, E=198.10^{9} \mathrm{~Pa}, \rho=7,850 \mathrm{~kg} / \mathrm{m}^{3}, v=0.3$ and $\alpha_{T}=17.3 \times 10^{-6} \mathrm{~K}^{-1}$. This very thin plate is subjected to harmonic loading with frequency of excitation $\omega_{h}=172 \mathrm{rad} / \mathrm{s}\left(0.7 \omega_{1,1}\right)$ and amplitude $p=0.3 \mathrm{~N}$ The time domain response of the plate center is shown in Fig. 2. The amplitudes of oscillations are very close to the ones shown in Fig. 9 in [1], so the verification of the present results is satisfactory.

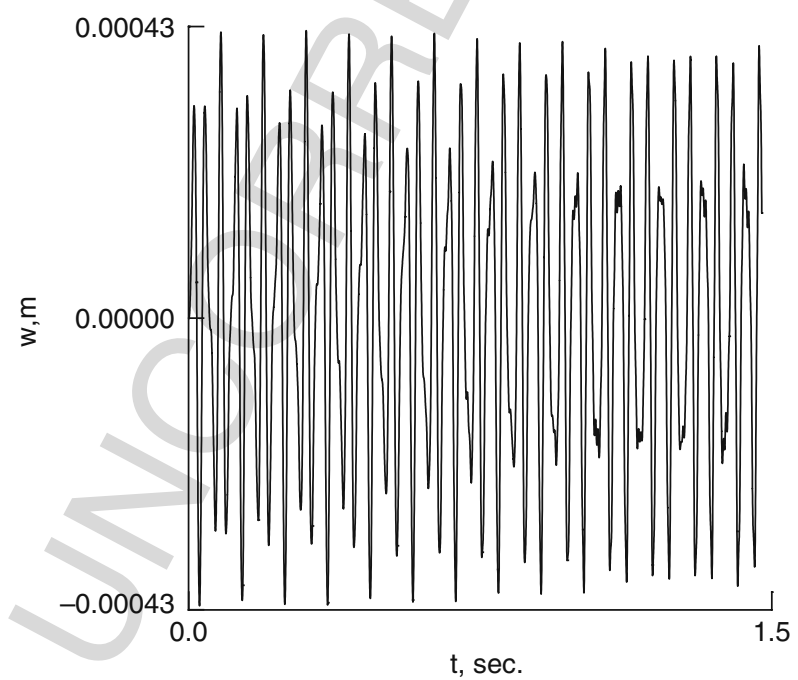

Fig. 2 Vibration response at the plate centre $\left(\omega_{h}=172 \mathrm{rad} / \mathrm{s}, p=0.3 \mathrm{~N}\right)$ 


\section{Damage Detections in Nonlinear Vibrating Thermally Loaded Plates}

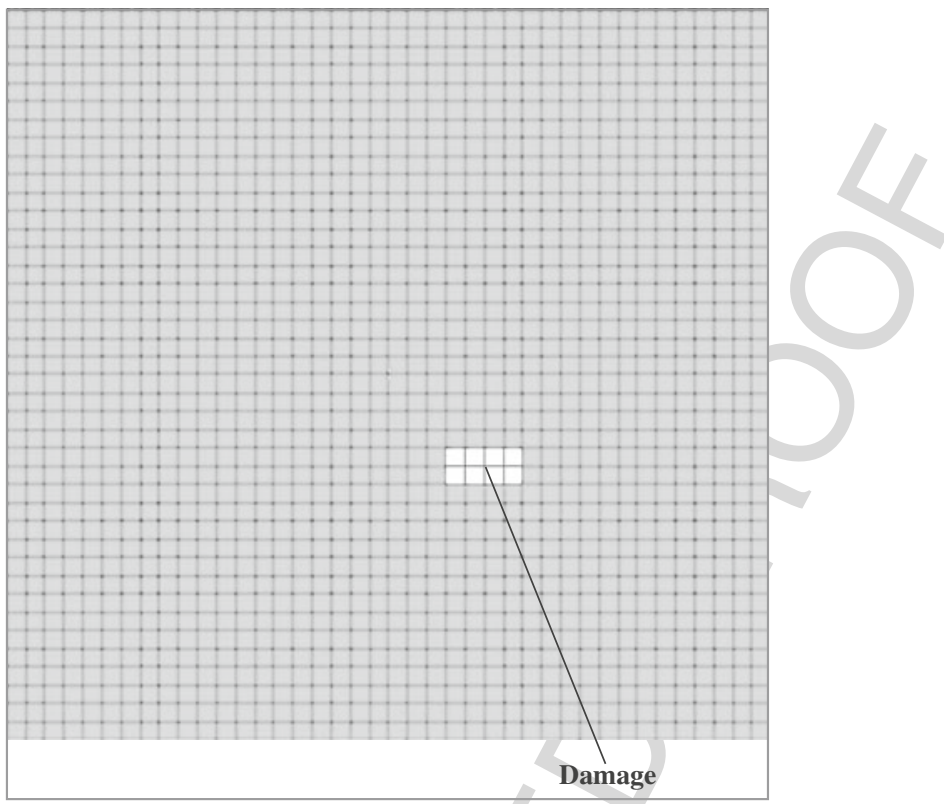

Fig. 3 Finite element discretization and damaged area (white colour) of the plate

Then the same plate but with increased thickness $h=0.0005 \mathrm{~m}$ (case B from [1]) was subjected to thermal and dynamic loading. For this plate two cases were considered: (1) undamaged plate and (2) plate with reduced thickness in a small part of the plate - the white area from the plate shown in Fig. 3.

It was shown in [1] that the buckling temperature for this plate is $\Delta T=0.9 \mathrm{~K}$. It is clear that the attempt to inspect such a plate for damage without considering the temperature changes is condemned to fail.

In Fig. 4 the time-history diagrams of the healthy and the damaged plate subjected to a harmonic loading $p=0.9 \mathrm{~N}$ applied in the plate centre with frequency of excitation $\omega_{h}=319 \mathrm{rad} / \mathrm{s}$. $\left(\omega_{1,1}=455.6 \mathrm{rad} / \mathrm{s}\right)$ are shown. Inspecting the time history it is visible that at the beginning the introduced small defect doesn't influence essentially the response of the plate but small changes in the eigen frequencies and modes lead to phase shift and the differences between the two responses increase with time. The phase shift can be clearly seen on the small figure in Fig. 4 where a short interval from the response is shown. The Poincare maps of the responses of the healthy and the damaged plate in the plate centre (Fig. 5a) and in the centre of the defect are shown in (Fig. 5b), respectively. The Poincaré plots shown are obtained as a projection of $(w, \dot{w})$ at moments $t$, where $t$ is a multiple of the period $T=2 \pi / \omega_{h}$. The damage doesn't change essentially the form of the Poincaré plot. As can be expected the difference between the two responses is larger at the points with reduced thickness. A contour plot of the damage index obtained by using Eq. (18) is plotted in Fig. 6 where a threshold value $T^{d}=0.06$ is used. The contour plot is a graphical technique for representing a 3-dimensional surface by plotting 


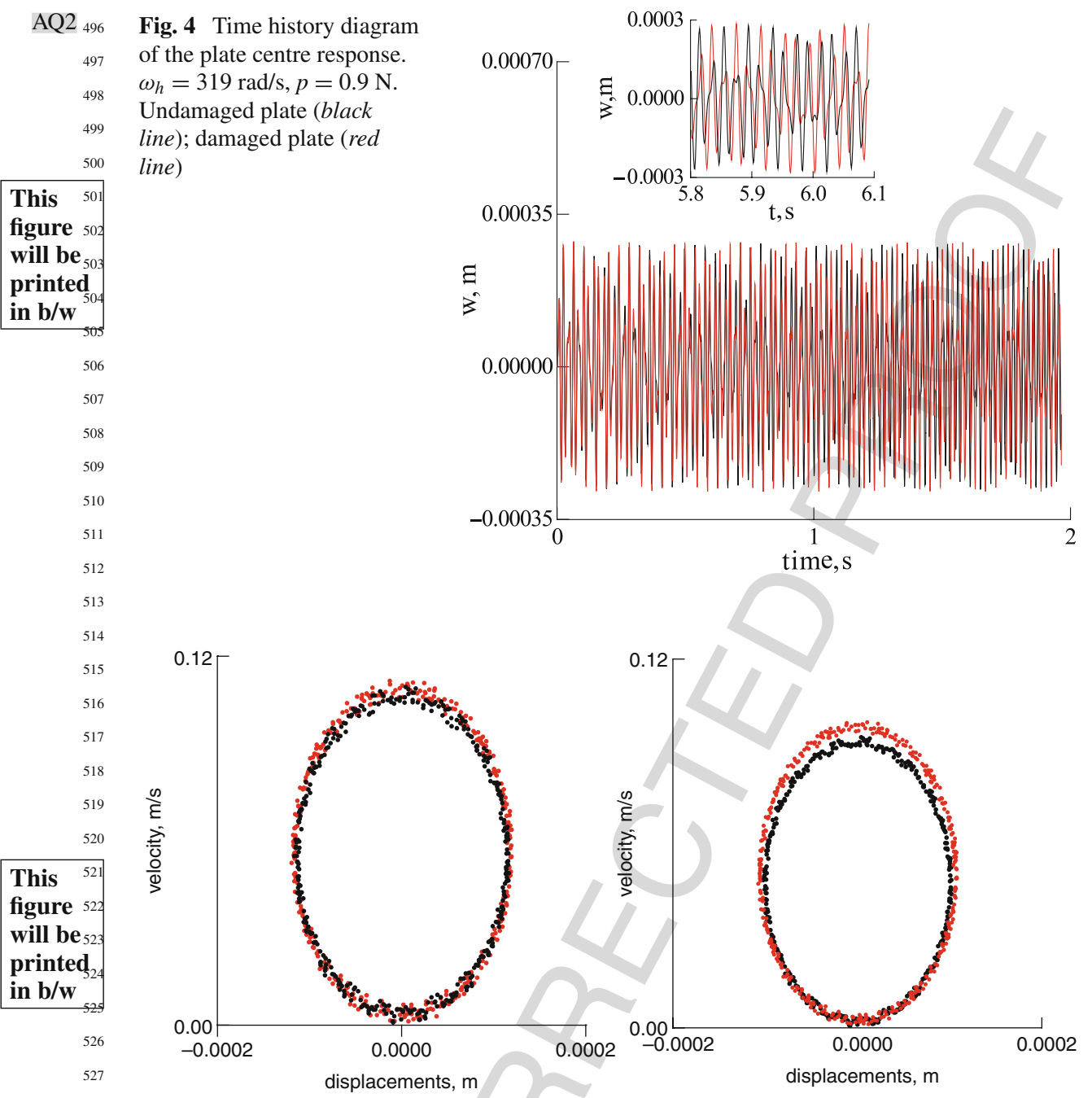

Fig. 5 (a) Poincaré map at the plate centre. Undamaged plate (black dots); damaged plate (red dots). (b) Poincaré map at the centre of the defect. Undamaged plate (black dots); damaged plate (red dots)

constant $z$ slices, called contours, on a 2-dimensional plane. That is, for a given value of $z$, lines are drawn that connect the $(x, y)$ coordinates which correspond to this particular value of $z$. The contour plot is compared to the FE model of the plate where the damaged area is coloured in white. As can be seen the damage criterion in this case works quite well and predicts rather precisely the damage location despite of the fact that the damage indexes have low values.

Then the same plates were considered at elevated temperature namely $\Delta T=$ $0.7 \mathrm{~K}$. This temperature leads to increased amplitudes of vibrations of the plates 
Damage Detections in Nonlinear Vibrating Thermally Loaded Plates
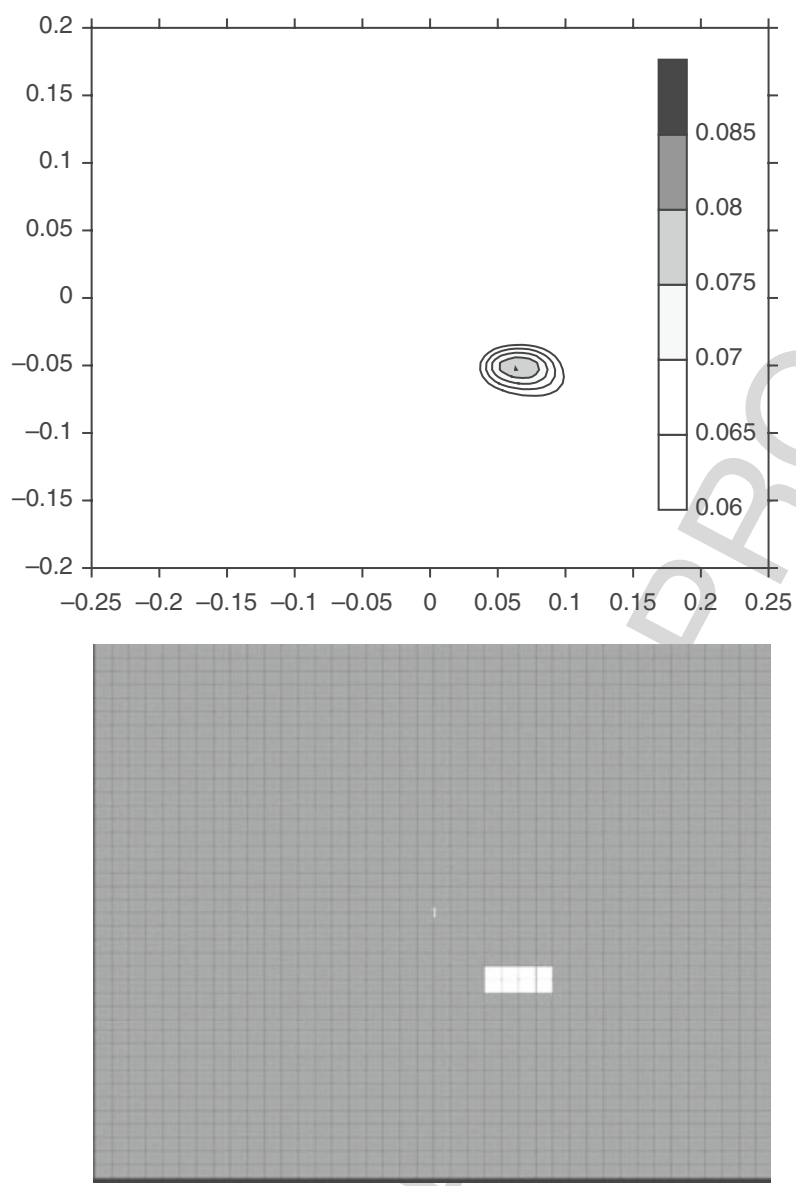

Fig. 6 Contour map of the damage index (unheated plate) and comparison with the damage location

(see Fig. 7). Again, the differences in the plate history diagrams are visible but they are not very large in the beginning of the time histories. However the Poincaré plots for the damaged and the undamaged plate have very different shapes, as can be seen from Fig. 8. This phenomenon may indicate that for these loading parameters the dynamic system changes its position in the basin of attractions moving from one region to another. This observation agrees with the fact that the plate buckles at $\Delta T=0.9 \mathrm{~K}$ [1]. The shapes of the Poincaré plots at the damaged nodes are similar. Obviously, in such case the damage criterion (20) is not appropriate and doesn't give satisfactory results for the damage location (not shown here). As can be expected neglecting the temperature influence is impossible for the damage detection purpose and leads to wrong results.

The second numerical example concerns a thicker rectangular plate with the following geometrical and material properties: $a=10 \mathrm{~m}, b=2.5 \mathrm{~m}, h=0.05 \mathrm{~m}$, 


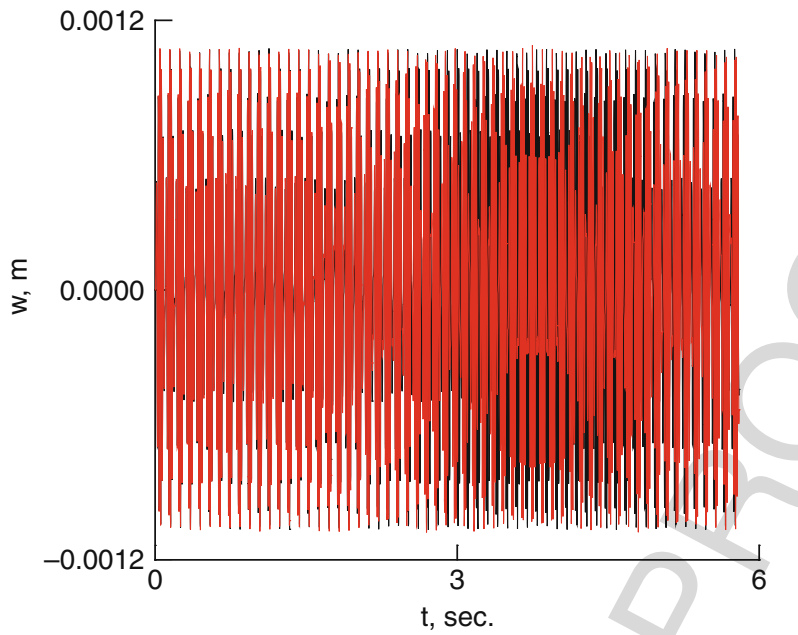

Fig. 7 Time history of the thermally loaded plate. Undamaged plate (black line); damaged plate (red line). $\Delta T=0.7 \mathrm{~K}$

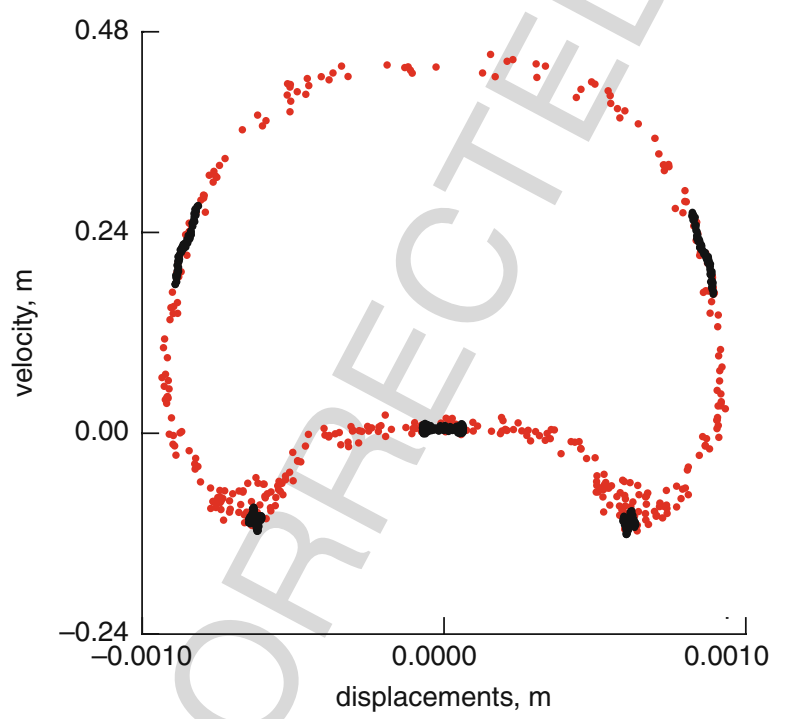

Fig. 8 Poincaré map of the response of the plate centre of heated undamaged (black dots) and damaged (red dots) plates. $\Delta T=0.7 \mathrm{~K}$

Young modulus $E=7.10^{10} \mathrm{~N} / \mathrm{m}^{2}$, Poison ratio $v=0.34$, density $\rho=2,778 \mathrm{~kg} / \mathrm{m}^{3}$. The damping coefficient $c_{1}=c_{2} \frac{12}{h^{2}}$ in Eq. (8) was chosen to be $0.00075 \frac{\mathrm{N} \mathrm{s}}{\mathrm{m}^{3}}$. The finite element discretization and the damage area are shown in Fig. 9. Again, the damaged area has a thickness $h_{\text {damaged }}=h / 2$. The plate is fully clamped and the applied harmonic load $p=500 \mathrm{~N}$ is uniformly distributed over the whole plate 


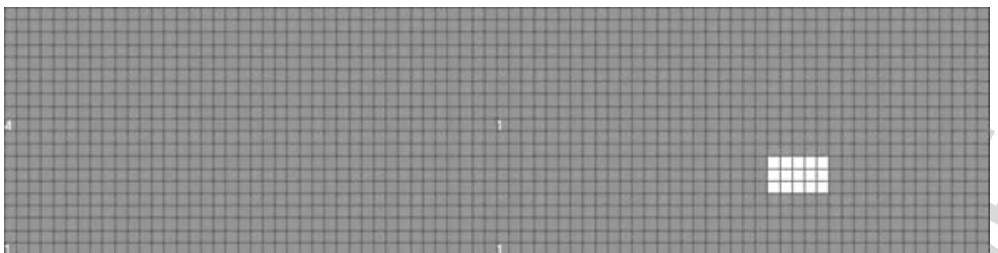

Fig. 9 Finite element mesh of the plate with defect

surface. The time history diagrams of the plate centre of the plate with a defect and without defect are shown in Fig. 10. The same time history diagrams but in the case of elevated temperatures of the plates are shown in Fig. 11. The excitation frequency is $260 \mathrm{rad} / \mathrm{s}$, which is only $7 \%$ less than the first eigen frequency of the healthy plate. A strong beating can be observed in the responses of the healthy and damaged plates. The phase of the response of the damaged plate shifts and the difference between the responses increases with the time. The same conclusion applies in the case of the rectangular plate at elevated temperature. The elevated temperature leads to larger values of the vibration amplitude. Again, the differences between the Poincaré plots of the heated and unheated plates are largest for the points from the damaged areas (see Fig. 12a-c). Accordingly, the damage indexes corresponding to the damaged area have the biggest values, which gives the possibility to locate the damage. The contour plots of $I_{i}^{d}$ corresponding to three different temperatures are shown in Fig. 13. It can be seen that the damage location is predicted very precisely in the case of the unheated plate as well as in the

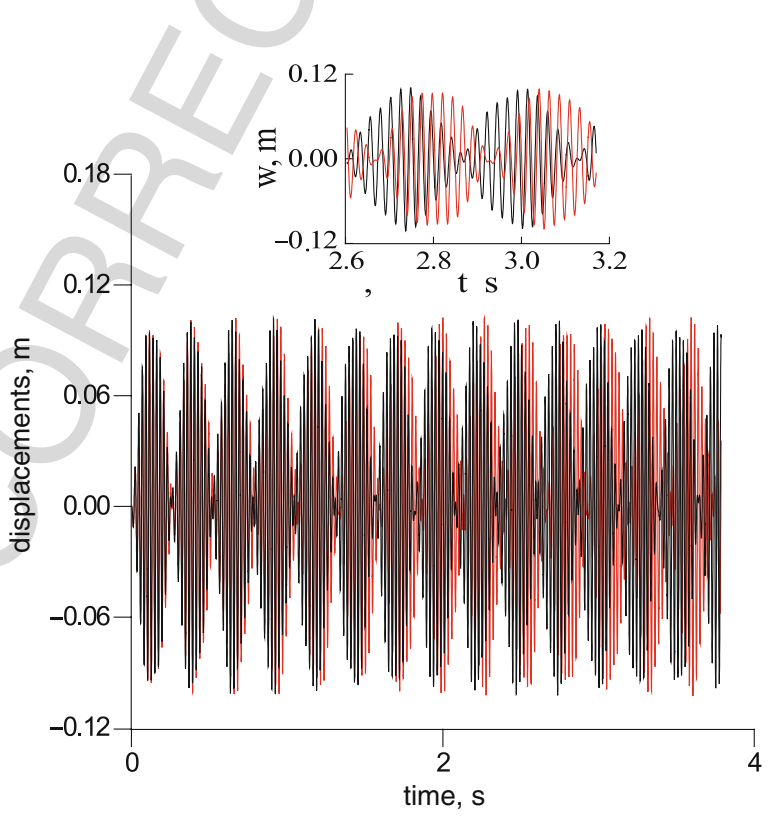

This figure will be printed in $\mathbf{b} / \mathbf{w}$
Fig. 10 Time history diagram of the plate centre , $p=500 \mathrm{~N}, \omega_{h}=260 \mathrm{rad} / \mathrm{s}$

time, $s$ 

will be printed in $\mathbf{b} / \mathbf{w}^{70}$
Fig. 11 Time history diagram of the plate center of heated plate, $p=500 \mathrm{~N}$, $\omega_{h}=260 \mathrm{rad} / \mathrm{s}, \Delta T=50 \mathrm{~K}$

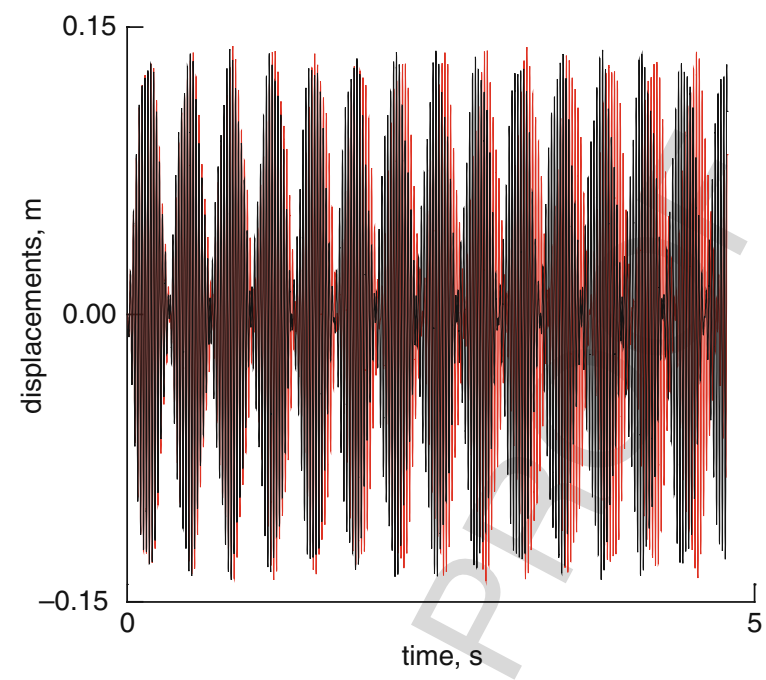

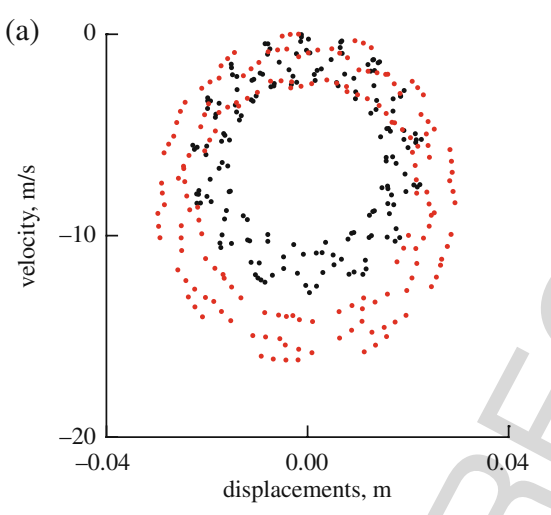

(b) 0

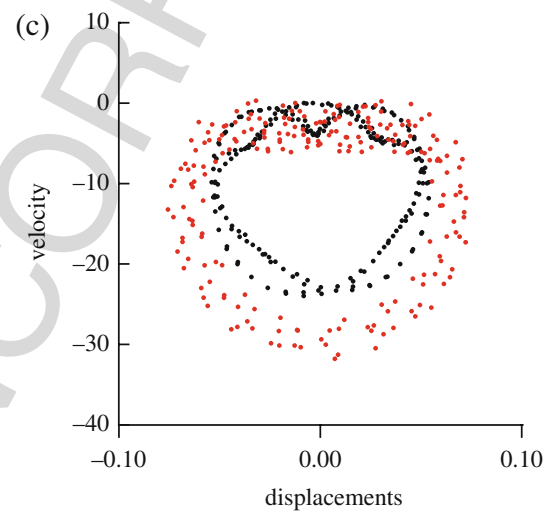

Fig. 12 Poincaré map at the centre of the defect for: (a) unheated plate, (b) heated plate $\Delta T$. $=$ $50 \mathrm{~K}$, (c) heated plate $-\Delta T=100 \mathrm{~K}$. Undamaged plate (black dots); damaged plate (red dots) 
Damage Detections in Nonlinear Vibrating Thermally Loaded Plates

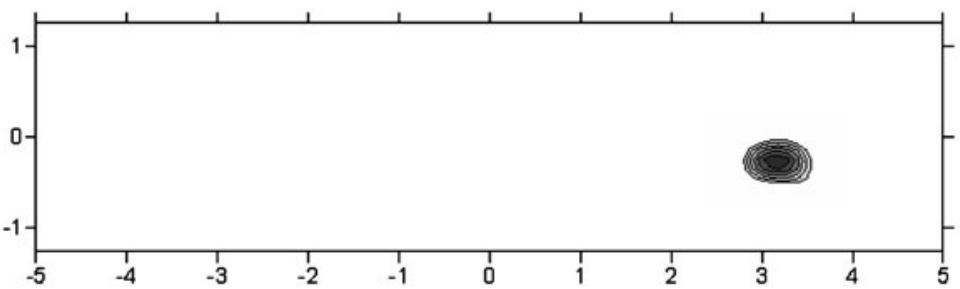

(a) $\Delta \mathrm{T}=\mathrm{OK}$

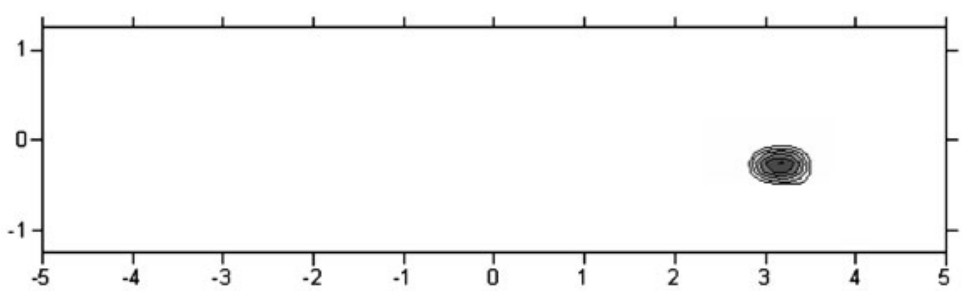

(b) $\Delta \mathrm{T}=50 \mathrm{~K}$

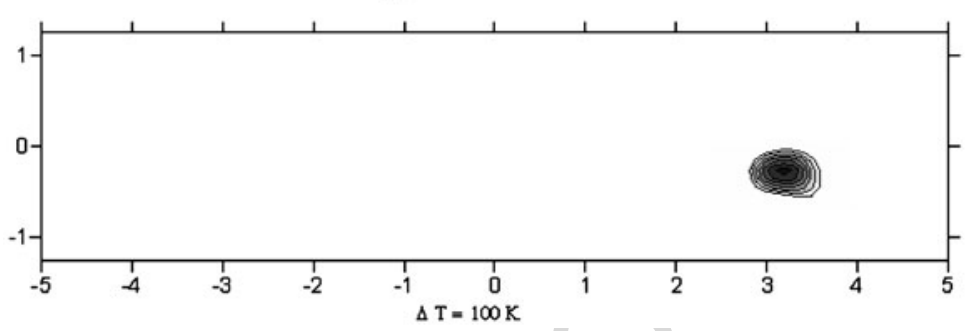

Fig. 13 Contour maps of the damage index for unheated and heated rectangular plate with damage

cases of the heated plate with two different temperatures $\Delta T=50 \mathrm{~K}$ and $\Delta T=$ $100 \mathrm{~K}$. The threshold value $T^{d}$ is set to 0.28 for all cases and the maximal value of $I^{d}$ is almost the same $\left(I^{d}=0.4\right.$ for $\Delta T=0, \Delta T=50 \mathrm{~K}$ and $I^{d}=0.42$ for $\Delta T=100 \mathrm{~K})$.

If, however one calculates, for example the damage index of the healthy unheated plate and the one for the damaged but heated plate then the damage location cannot be predicted precisely. This is due to the temperature change which is not taken into account for the healthy plate. The vibration responses of the healthy and the damaged plates should be compared for the same temperatures.

\section{Conclusions}

In this paper the computed time domain vibration responses are used to analyse the dynamic behaviour of plates in the intact condition and in the case when defects are present taking into account the temperature changes. A damage assessment method 
is suggested which is based on the phase space representation of the time domain nonlinear vibration response of the plate and uses the analysis of its Poincaré map. It has been demonstrated that damage as well as elevated temperatures can influence substantially the time domain response of the plate and its Poincaré maps. It can be concluded that: 1) The influence of the temperature changes is essential and can change substantially the nonlinear dynamic response of the plate and this is why temperature changes should be taken into account when developing a damage assessment procedure; 2) Temperature loadings which lead to either buckling or chaotic behaviour of the plate, might render the damage criterion suggested by Eqs. (18), (19) and (20) inappropriate. This is because even small damage, resulting in stiffness reduction of the plate, could lead to dramatic changes in the Poincaré maps of the response and consequently to unreliable results.

The potential, the sensitivity and the applicability of the developed method still have to be tested for real measurements and for more structures, defects and loading conditions.

Acknowledgments The first author wishes to thank the Bulgarian Research Fund for the partial support through grant $\mathrm{TN}-1518 / 2005$.

\section{References}

1. M. Amabili, S. Carra, Thermal effects on geometrically nonlinear vibrations of rectangular plates with fixed edges. J. Sound Vib. 321, 936-954 (2009)

2. E. Manoach, Dynamic large deflection analysis of elastic-plastic Mindlin circular plates. Int. J. Nonlinear Mech. 29, 723-735 (1994)

3. E. Parloo, P. Verboven, P. Guillaume, M. van Overmeire, Autonomous structural health monitoring - Part II: Vibration-based in-operation damage assessment. Mech. Syst. Signal Process. 16, 659-675 (2002)

4. P.F. Rizos, N. Aspragathos, A.D. Dimarogonas, Identification of crack location and magnitude in a cantilevered beam from the vibration modes. J. Sound Vib. 138, 381-388 (1990)

5. I. Trendafilova, E. Manoach, M.P. Cartmell, W. Ostachowicz, A. Zak, An investigation on damage detection in aircrafts panels using nonlinear time series analysis. Key Eng. Mater. 347, 213-218 (2007)

6. I. Trendafilova, E. Manoach, Vibration based damage detection in plates by using time series analysis. Mech. Syst. Signal Process. 22, 1092-1106 (2008)

7. P. Verboven, E. Parloo, P. Guillaume, M. van Overmeire, Autonomous structural health monitoring - Part I: Modal parameter estimation and tracking. Mech. Syst. Signal Process. 16, 637-657 (2002)

8. Y. Zou, L. Tong, G.P. Steven, Vibration based model-dependent damage (delamination) identification and health monitoring for composite structures - A review. J. Sound Vib. 230, 357-378 (2000)

9. H.T. Banks, D.J. Inman, D.J. Leo, Y. Wang, An experimentally validated damage detection theory in smart structures. J. Sound Vib. 191, 859-880 (1996)

10. L. Moniz, J.M. Nichols, C.J. Nichols, M. Seaver, S.T. Trickey, M.D. Todd, L.M. Pecora, L.N. Virgin, A multivariate, attractor-based approach to structural health monitoring. J. Sound Vib. 283, 295-310 (2005)

11. P. Ribeiro, E. Manoach, The effect of temperature on the large amplitude vibrations of curved beams. J. Sound Vib. 285, 1093-1107 (2005) 
Damage Detections in Nonlinear Vibrating Thermally Loaded Plates

12. E. Manoach, in Dynamic Large Deflection Analysis of Elastic-Plastic Beams and Plates, ed. by N.S. Ferguson, H.F. Wolfe, M.A. Ferman, S.A. Rizzi. Proceedings of 7th International Conference Recent Advances on Structural Dynamics, vol. 1 (The Institute of Sound and Vibration Research University, Southampton, 2000), pp. 389-400

13. J. Cattarius, D.J. Inman, Time domain analysis for damage detection in smart structures. Mech. Syst. Signal Process. 11, 409-423 (1997)

14. B.I. Epureanu, L.S. Tang, M.P. Päıdoussis, Exploiting chaotic dynamics for detecting parametric variations in aeroelastic systems. AIAA J. 42, 728-735 (2004)

15. E. Manoach, in Coupled, Large Amplitude Vibrations of Circular Plates Subjected to Mechanical and Thermal Loading. ed. by D.H. van Campen, M.D. Lazurko, W.P.J.M. van den Oever. Proceedings of ENOC-2005, Fifth EUROMECH Nonlinear Dynamic Conference, Eindhoven, The Netherlands (2005), pp. 2548-2557

16. A.R. Kukreti, H.I. Issa, Dynamic analysis of nonlinear structures by pseudo-normal mode superposition method. Comput. Struct. 19, 653-663 (1984)

17. P. Ribeiro, Thermally induced transitions to chaos in plate vibrations. J. Sound Vib. 299, 314-330 (2007)

18. Thorton, E.A, Thermal Structures for Aerospace Applications, AIAA Education Series (AIAA: Washington, DC, 1996)

19. M. Todd, J.M. Nichols, L.M. Pecora, L. Virgin, Vibration-based damage assessment utilizing state space geometry changes: Local attractor variance ratio. Smart Mater. Struct. 10, 1000-1008 (2001)

20. E. Manoach, P. Ribeiro, Coupled, thermoelastic, large amplitude vibrations of Timoshenko beams. Int. J. Mech. Sci. 46, 1589-1606 (2004)

21. E. Manoach, I. Trendafilova, Large amplitude vibrations and damage detection of rectangular plates. J. Sound Vib. 315, 591-606 (2008) 


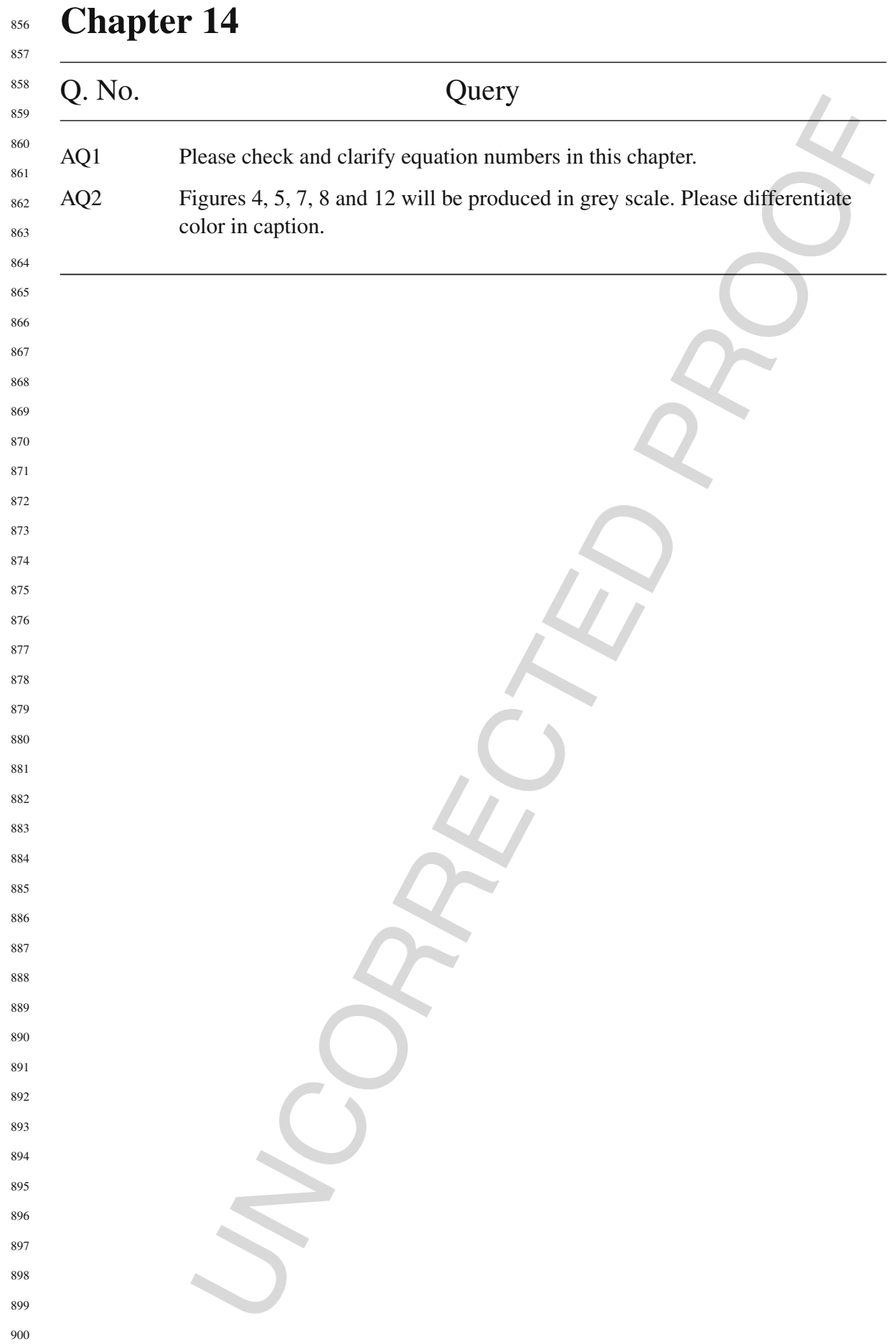

